Paediatr. Paedolog. 2019 · 54 (Suppl 1):S65-S70 https://doi.org/10.1007/s00608-019-0664-z Online publiziert: 21. März 2019

(c) Der/die Autor(en) 2019

\author{
Thomas Lion ${ }^{1,2,3}$ \\ 'Wien, Österreich \\ ${ }^{2}$ St. Anna Kinderspital, Wien, Österreich \\ ${ }^{3}$ Medizinische Universität Wien, Wien, Österreich
}

\title{
Supervision im ärztlichen Bereich - eine super Vision?
}

\begin{abstract}
Während Supervision und Coaching insbesondere bei Führungskräften in verschiedenen beruflichen Kontexten selbstverständliche Reflexions- und Beratungsformate darstellen, ist die Akzeptanz im ärztlichen Bereich, zumindest in den somatischen Fächern, vergleichsweise noch gering. Das ist überraschend, weil die Herausforderungen, Belastungen und Spannungsfelder im beruflichen Alltag von Ärztinnen und Ärzten vielfältige Themen hervorbringen, die in diesem Rahmen zielführend bearbeitet werden könnten. Es ist daher interessant Überlegungen anzustellen, worauf die ablehnende Haltung gegenüber diesen Beratungsformaten beruhen könnte.
\end{abstract}

\section{Was ist Supervision, was ist Coaching?}

Die Begriffe sind für jemanden, dem diese Beratungsformate nicht geläufig sind, nicht ganz glücklich gewählt, weil die Bezeichnungen Supervision und Supervisor, in Anlehnung an den angloamerikanischen Sprachgebrauch, die Interaktion mit einer vorgesetzten Person suggerieren könnten. Der Begriff Coaching ist in dieser Hinsicht eventuell anschlussfähiger, obwohl der Coach mit einem Trainer assoziiert wird, der üblicherweise bestimmt, was und vor allem wie etwas zu tun ist, also letztlich auch wieder eine Art Vorgesetztenfunktion ausübt. Keine der Bezeichnungen ist daher intuitiv mit einem begleitenden Reflexionsprozess auf Augenhöhe assoziiert, sodass für die Ak- zeptanzentsprechende Informationen im Vorfeld erforderlich sind.

Laut Definition der österreichischen Gesellschaft für Supervision und Coaching ist die Supervision eine „professionelle Beratungsmethode für alle beruflichen Herausforderungen von Einzelpersonen, Teams und Organisationen und unterstützt dabei, berufliche Handlungen zielgerichtet, effizient und erfolgreich zu gestalten. Sie dient der Unterstützung von Menschen in beruflichen Angelegenheiten, indem Fragen und Themen, die sich aus den Anforderungen des Berufs ergeben, reflektiert, geklärt und alternative Handlungsmöglichkeiten erarbeitet werden. "Coaching ist hingegen als eine spezielle Form von Supervision definiert, die sich primär an Personen mit Führungsaufgaben richtet. Während beim Coaching primär eine themenspezifische und handlungsrelevante Unterstützung sowie die Vermittlung von Fähigkeiten für den beruflichen Alltag im Vordergrund stehen, liegt bei der Supervision der Reflexionsaspekt stärker im Fokus. In • Tab. 1 sind einige Beispiele für supervisionsrelevante Themenbereiche angeführt.

\section{Besteht im ärztlichen Bereich ein Bedarf an Supervision und Coaching?}

Wenn man die Website des Wiener Krankenanstaltenverbundes (http://www. wienkav.at) besucht, findet man die Information, dass flächendeckende Supervision, Krisenmanagement und Coaching als Burn-out-Unterstützungsmaßnahmen angeboten werden. Supervision im Krankenhaus wird aber derzeit offenbar fast ausschließlich für Teams im Pflegebereich durchgeführt [1]. Auf der Website der niederösterreichischen Ärztekammer (https://cms.arztnoe.at) kann man folgende Statements lesen: „Supervision für Ärztinnen und Ärzte ist ein unverzichtbares Element in der ärztlichen Ausbildung und in der Fortund Weiterbildung. Sie ist notwendig im Zusammenhang mit Qualitätssicherung und eigener Psychohygiene. Supervision für Ärzte ist deshalb auch in der Ärzteausbildungsordnung verankert.“ Auch die Tatsache, dass Ärztinnen und Ärzte für die Inanspruchnahme von Supervision unter bestimmten Voraussetzungen Fortbildungspunkte im Diplom-Fortbildungs-Programm sammeln können, unterstreicht, dass die Bedeutung dieses Beratungsformats von institutioneller Seite anerkannt und gefördert wird.

\section{Studien über Supervision und Coaching im ärztlichen Bereich}

$\mathrm{Zu}$ diesem Thema gibt es, zumindest im deutschsprachigen Raum, wenig Literatur, aber die vorliegenden Studien [2, 3] kamen zu dem Schluss, dass im ärztlichen Bereich im Allgemeinen und in der ärztlichen Führungsebene im Besonderen - außerhalb der Fächer Psychiatrie und Psychosomatik - häufig eine skeptische bis ablehnende Haltung gegenüber dem Format Supervision vorherrscht. Innerhalb der somatischen Fachbereiche ist die Teamsupervision etwa in der pädiatrischen Onkologie oder in der Palliativmedizin gut verankert, und man könnte vielleicht annehmen, dass die Akzeptanz im pädiatrischen Bereich relativ hoch sein könnte, aber entsprechende Studiendaten fehlen. Die angeführten Studien wei- 


Tab. 1 Was bietet Supervision (Beispiele)
Reflexions- und Entscheidungshilfe (bei aktuellen Anlässen)
Unterstützung in herausfordernden oder belastenden Arbeitssituationen
Unterstützung bei Konflikten
Klärung und Gestaltung von Aufgaben, Aufträgen, Funktionen und Rollen
Begleitung bei Veränderungsprozessen und deren Bewältigung
Innovative Lösungen bei neuen Herausforderungen
Mobbing- und Burn-out-Prophylaxe
Beratung in Führungs- und Managementaufgaben
Impulse zur Gestaltung der Führungsrolle
Entwicklung der persönlichen Performance (z. B. bei Präsentationen)

sen jedenfalls daraufhin, dass die Annahme von Supervision und Coaching umso geringer wird, je näher man den chirurgischen Fächern kommt. Wenn man der Frage nachgeht, woher die ablehnende Haltung im ärztlichen Bereich kommen könnte, liefern Narrative aus der griechisch-römischen Antike interessante Ansatzpunkte für die Wurzeln des ärztlichen Habitus.

\section{Kulturhistorische Wurzeln des ärztlichen Selbstverständnisses in der Antike}

Die Perzeption des ärztlichen Berufes bei Homer ist mit seiner Überzeugung von der großen Bedeutung der Heilkunst verknüpft. In der Ilias beschreibt er den Helden Machaon, Sohn des Heilgottes Asklepios und Enkel von Apollon, als unvergleichbaren Arzt, der den verletzten König Menelaos medizinisch versorgt. Ein Schlüsselsatz findet sich im elften Gesang der Ilias. Hier ersucht Idomeneus (König von Kreta und einer der großen Helden der Achäer vor Troja) Nestor (Herrscher von Pylos und wichtiger Berater Agamemnons), den an der Schulter verwundeten Arzt Machaon aus der Schlacht zu retten und zu den Schiffen zu bringen. Dabei sagt er folgende Worte: „[...] ia

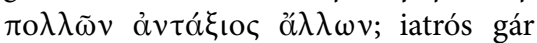
anér pollón antaxios allón; denn ein Arzt ist ein Mann, der wert ist (so viel zählt wie) viele andere (zusammen)“ (Ilias XI: 514). Die große Wertschätzung, die im antiken Griechenland den Ärzten entgegengebracht wurde, konnte kaum eindrucksvoller zum Ausdruck gebracht werden, und dieser Satz stand übrigens in großen Lettern in einem Hörsaal der Medizinischen Universität Wien.

Die bedeutendste Rolle in der griechischen Medizin der Antike spielte Hippokrates (460-377 v. u. Z.), der als prominentester Vertreter der medizinischen Schule von Kos und Vater der modernen Medizin gilt. Das berühmte Corpus Hippocraticum enthält eine Sammlung von etwa 70 frühen medizinischen Werken des antiken Griechenland, die in enger Verbindung mit Hippokrates und seinen Schülern stehen. Bekannt ist Hippokrates auch als Urheber des hippokratischen Eides, aus dessen Existenz hervorgeht, dass die damalige Medizin von einer Gruppe professioneller Ärzte praktiziert wurde, die an einen strengen ethischen Kodex gebunden waren. Dieser Eid, der (mit zeitgemäßen Adaptierungen der World Medical Association) im Rahmen der Promotion von Ärztinnen und Ärzten an vielen Universitäten abgelegt wird, ist bis heute von wesentlicher Bedeutung, denn er gilt als erste grundlegende Formulierung ärztlicher Ethik. Überliefert ist auch der Hippokrates zugeschriebene Ausspruch, dass ein philosophischer Arzt ein wahrhaft göttergleicher Mann ist (sinngemäß genauer übersetzt: ein Arzt, der - im sokratischen Sinn - ein Weiser ist, das ist ein göttergleicher Mann; [4]). Hier wird die Bedeutung der Verknüpfung zwischen Philosophie (Weisheit, Wissen) und Medizin hervorgehoben. Wenn man den Ausdruck göttergleich auch als mächtig versteht, wäre im Sinne Foucaults (s. unten) die zwingende Verbindung zwischen Wissen und Macht in diesem Satz mit enthalten. Im Übrigen war auch Hippokrates der Überlieferung nach göttlicher Herkunft, denn er wird väterlicherseits als 18. Enkel von Asklepios und mütterlicherseits als 20. Enkel von Herakles beschrieben. Haben also die späteren Götter in Weiß hier eine kulturhistorische Vorlage?

Die Hippokratiker lehrten Heilkunde als Kunst und Überschau über das Geschehen in den Krankheitstagen und widmeten der Natur des Einzelnen die größte Aufmerksamkeit. Vom Arzt wurde nicht nur Ausbildung, sondern auch künstlerische Begabung erwartet. Nach Hippokrates behandelt der wissenschaftliche Arzt die gleichen Krankheiten bei verschiedenen Menschen immer gleich, weil er sich nach der Krankheit und nicht nach dem kranken Menschen richtet, während der künstlerisch eingestellte Arzt aus seiner Krankheitslehre heraus die allgemeinen Behandlungsregeln kennt und von Fall zu Fall behandelt, je nach den im Augenblick vorliegenden Besonderheiten (man könnte es als eine Vorwegnahme der heute besonders aktuellen personalisierten Medizin sehen). Dabei wird er, die künstlerische Begabung vorausgesetzt, durch seine Intuition unterstützt. Die ärztliche Selbstwahrnehmung als Künstler könnte vielleicht hier ihre Wurzeln haben. Dabei könnte paradoxerweise eine irreführende Übersetzung eine wesentliche Rolle gespielt haben, denn der mit Kunst übersetzte Ausdruck lautet im altgrie-

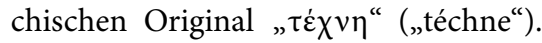
„Téchne“ ist ein Begriff, der laut Wikipedia in europäisch geprägter Philosophie bis heute für das Verständnis von Kunst, Wissenschaft und Technik bedeutend ist. Der Ursprung der Auffassung von "téchne“ ist aber aus der Ilias rekonstruierbar. Dort bedeutete „technē" das Können der Handwerker und wird damit als Mittel zur planmäßigen Erreichung eines Ziels gesehen. Wurde die Medizin in der Antike daher primär als Handwerk gesehen und entspringt die Selbstwahrnehmung als Künstler einer narzisstisch angehauchten (Fehl-)Interpretation? Durch längere Kontakte mit der griechischen Kultur und die schließliche Eroberung Griechenlands übernahmen die Römer zahlreiche griechische medizinische Ideen und letztlich auch die hippokratische Medizin. Diese Akzeptanz führte zur Ausbreitung der griechischen medizinischen Theorien im ge- 
samten Römischen Reich und damit in einem großen Teil des Westens. Das Wort Medizin leitet sich bekanntlich vom lateinischen Verb „mederi“ (heilen, helfen) $a b$, und aus der altgriechischen Bezeich-

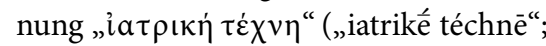
ärztliche Kunst oder ärztliches Handwerk) wurde im Lateinischen „ars medicina“, also doch ärztliche Kunst? Jedenfalls scheint sich das Selbstverständnis als Künstler viel stärker als jenes des Handwerkers in den ärztlichen Habitus projiziert zu haben.

Einflussreichster Gelehrter, der die hippokratische Tradition in Rom fortsetzte und erweiterte, war der griechische Arzt Galenos von Pergamon (etwa 130-207). Sämtliche hippokratischen und galenischen Texte waren aber nach dem Zusammenbruch des Weströmischen Reiches im lateinischen Westen im Frühmittelalter verschollen, im Oströmischen Reich (Byzanz) wurden sie jedoch weiter studiert und befolgt. Nach 750 u. Z. übersetzten insbesondere die muslimischen Araber Galens Werke und übernahmen später die hippokratisch-galenische Tradition, bis sie diese schließlich eigenständig unter besonderem Einfluss Avicennas (etwa 980-1037) erweiterten. Ab Ende des 11. Jahrhunderts kehrte die hippokratisch-galenische Tradition mit zahlreichen arabischen Übersetzungen und einigen griechischen Originaltexten in den lateinischen Westen zurück. In der Renaissance wurden Übersetzungen von Galen und Hippokrates direkt aus dem Griechischen aus neu zugänglichen byzantinischen Handschriften angefertigt. Galens Einfluss war über viele Jahrhunderte so groß, dass die Gelehrten oftmals ihre Ergebnisse, die mit den galenischen Prinzipien nicht in Einklang waren, dennoch in das galenische Modell gezwängt haben. Der Einfluss hippokratisch-galenischer Tradition wurde aber endgültig erst im 19. Jahrhundert eingedämmt, als etwa die mikroskopischen Studien Louis Pasteurs, Robert Kochs und anderer nachwiesen, dass bestimmte Krankheiten nicht durch ein Ungleichgewicht der 4 Körpersäfte, sondern durch Mikroorganismen wie Bakterien verursacht werden. Manche medizinische Begriffe, die wir bis heute verwenden,

Paediatr. Paedolog. 2019 · 54 (Suppl 1):S65-S70 https://doi.org/10.1007/s00608-019-0664-z (c) Der/die Autor(en) 2019

\section{T. Lion}

\section{Supervision im ärztlichen Bereich - eine super Vision?}

\section{Zusammenfassung}

Ärztinnen und Ärzte nehmen Supervision bzw. Coaching relativ wenig in Anspruch, obwohl die Reflexion beruflichen Handelns für ihre Arbeitswelten von hoher Relevanz wäre. Das könnte zum Teil an unzureichender Information über die Möglichkeiten und den Nutzen dieser Beratungsformate liegen. Zu einem erheblichen Teil jedoch, so die These dieser Arbeit, stellt das ärztliche Selbstverständnis einen in diesem Zusammenhang relevanten Unterschied zu anderen Berufsgruppen dar. Dem beruflichen Selbstbild liegt das Persistieren tradierter und verinnerlichter Narrative zugrunde, die ihren Ursprung in der Antike haben und die die Bedeutung des ärztlichen Berufes und die damit zusammenhängende Stellung in der Gesellschaft wesentlich geprägt haben. Die kulturhistorischen Einflüsse, die insbesondere auf Homer, Hippokrates und Galen zurückgehen, haben sich tief in die Matrix des ärztlichen Bewusstseins eingeschrieben. Philosophische Arbeiten von Michel Foucault und Pierre Bourdieu liefern mit den von innen geprägten Begriffen der Kontinuität kollektiver Erfahrung, des Habitus und der inkorporierten Geschichte Erklärungs- und Interpretationsvorlagen für die Genese des ärztlichen Selbstverständnisses.

\section{Schlüsselwörter}

Coaching · Habitus · Inkorporierte Geschichte . Kontinuität kollektiver Erfahrung · Bio-Macht

\section{Supervision in the Field of Medicine-a Super Vision?}

\section{Abstract}

Physicians utilize supervision or coaching relatively infrequently, despite the fact that reflection on professional actions is highly relevant in their working context. This may be at least partly attributable to the lack of information on the opportunities and benefits of these consulting formats. To a significant extent, however, according to the hypothesis of this work, the self-image of physicians represents a relevant difference to other professions in this context. The basis for the professional self-perception is provided by the persistence of conveyed and internalized narratives rooted in ancient times, which had a major impact on the prominence and on the resulting societal status of the medical profession. The cultural-historical influences, based particularly on Homer, Hippocrates, and Galen, became deeply engraved in the matrix of the self-awareness of physicians. The philosophical work of Michel Foucault and Pierre Bourdieu, and important terms coined by these authors, including continuity of collective experience, habitus, and incorporated history, provide the basis for the understanding and the interpretation of how the physicians' self-image was shaped.

\section{Keywords}

Coaching · Habitus · Incorporated history · Continuity of collective experience - Biopower lassen sich unmittelbar auf das antike Körpersäftekonzept zurückführen. Bekannte Beispiele wären etwa Melancho-

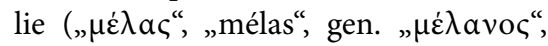

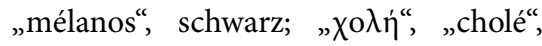
Galle, nach der Dominanz schwarzer Galle gemäß der Viersäftetheorie) oder

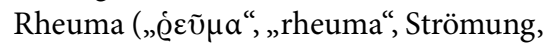
Fluss, da nach der Lehre der Körpersäfte geglaubt wurde, dass kalter Schleim vom Gehirn herab zu den Extremitäten fließt und die entsprechenden Beschwerden auslöst). Die Nachklänge aus der griechisch-römischen Antike sind in der
Medizin nach wie vor omnipräsent. Abgesehen von den Denkmälern, die den großen ärztlichen Vorbildern im wörtlichen und im übertragenen Sinn, etwa in Gestalt der Staue von Galen am Anatomischen Institut in Wien oder durch die Begriffe Galenik, „facies hippocratica“ bzw. den bereits erwähnten Hippokratischen Eid gesetzt wurden, sind die Bezeichnungen für diverse medizinische Fachrichtungen, Erkrankungen und diagnostische Verfahren aus griechischen, lateinischen oder aus beiden Sprachen zusammengesetzten Wörtern abgeleitet. 
Die im Krankenhaus übliche schriftliche Dokumentation des Krankheitsund Therapieverlaufs, der sogenannte Dekurs, stammt von „decursus morbi et therapiae“ oder der Vermerk „statim“ bzw. „stat“ (sofort) auf Zuweisungen $\mathrm{zu}$ dringenden Untersuchungen, sind weitere Beispiele, deren Auflistung man noch lange fortsetzen könnte, um die nach wie vor bestehende Omnipräsenz altgriechischer und lateinischer Begriffe in der Medizin zu dokumentieren, die als Echo einer lang zurückliegenden medizinischen Tradition nachhallen. Doch wie ist die Integration der angeführten kulturgeschichtlichen Aspekte in die Konstruktion des ärztlichen Selbstverständnisses vorstellbar? Wesentliche Grundlagen für dieses Verständnis lassen sich aus der geschichtsphilosophischen Forschung von Michel Foucault und der kultur- und sozialanthropologischen Forschung von Pierre Bourdieu ableiten.

\section{Philosophische Theorien zu ärztlicher Macht und dem ärztlichen Habitus}

Michel Foucault (1926-1984) stammte aus einer Ärztefamilie und befasste sich unter anderem auch mit medizinischen Themenbereichen, etwa in seinem Werk Die Geburt der Klinik: Eine Archäologie des ärztlichen Blicks [5], in dem die Entwicklung der Medizin und der Institution Klinik, mit der er insbesondere universitäre Lehrkrankenhäuser meinte, behandelt wird.

Der Zusammenhang zwischen Wissen und Macht ist ein grundlegendes Prinzip in der philosophischen Arbeit von Foucault. Er postulierte, dass diese Begriffe miteinander eng verflochten sind und einander reziprok bedingen. Beide Begriffe stellten für ihn Gegenstände eines fundamentalen Willensprinzips dar und er definierte den Willen zum Wissen auch als einen Willen zur Macht. Nach seiner Auffassung eröffnet umfassenderes und detaillierteres Wissen neue Möglichkeiten der Kontrolle, die umgekehrt den Erwerb weiteren Wissens ermöglicht. Macht und Wissen weisen daher zahlreiche Überlappungen auf [5-9]. Für Foucault war der Machtbegriff eine an sich positiv besetz- te Gestaltungsmöglichkeit, er sah ihn als Kraft, Dinge hervorzubringen und diese wachsen $\mathrm{zu}$ lassen, anstatt sie $\mathrm{zu}$ hemmen, beugen oder $\mathrm{zu}$ vernichten [7]. Letzteres impliziert aber auch die Option, Macht als Instrument zu gebrauchen bzw. potenziell $\mathrm{zu}$ missbrauchen, um Handlungen, Entwicklungen und (kreative) Prozesse zu behindern bzw. zu verhindern. Foucault postulierte, dass moderne Macht die gesamte Gesellschaft betrifft und als Mittel zur Disziplinierung und Kontrolle dient. Dabei betonte er, dass es für eine kapitalistisch orientierte Gesellschaft wesentlich ist, gut funktionierende Arbeitskräfte zu produzieren und dafür wäre körperliche Disziplinierung notwendig. Die Kontroll- und Disziplinierungstechniken erzeugen neues Wissen, da sie systematische Erkenntnisse über einzelne Menschen ermöglichen und diese dadurch $\mathrm{zu}$ beschreibbaren, analysierbaren und normierbaren Objekten machen. Die Disziplinierungsmacht liegt in komplexen sozialen Netzwerken verteilt und kann von Personen und Institutionen ausgeübt werden. Foucault befasste sich mit der Frage, wie bestimmtes Wissen Autorität erlangen kann und entwarf das Konzept der Bio-Macht, deren Ziel die Regulierung der Bevölkerung insbesondere im Hinblick auf die Gesundheit ist [9]. Die Bio-Macht und deren Techniken, die auf die gesamte Bevölkerung zielen, sind natürlich im Zusammenhang mit der Sicherstellung funktionierender Arbeitskräfte in kapitalistischen Gesellschaften zu verstehen, sodass die bestmögliche Nutzung der Ressource Mensch für den Staat gewährleistet wird. Die Auswirkungen der BioMacht betreffen die Gesamtgesellschaft gleichermaßen wie das Individuum und die Kontrolle wird als Angelegenheit des Staates dem Gesundheitswesen zugeordnet. Diese Überlegungen haben allerdings relevante Implikationen für die Rolle von Ärztinnen und Ärzten, denn in diesem Konzept treten sie als Exekutive auf, die quasi im staatlichen Auftrag als Wächterin über die Gesundheit auftritt. Ärztinnen und Ärzte üben somit einerseits Macht aus, da die Sorge um die Gesundheit einen wesentlichen Angriffspunkt für die Wirkung der BioMacht darstellt, unterliegen aber selbst der institutionellen Macht, für die sie lediglich eine exekutive Rolle ausführen. Darin liegt aber eine immanente Gefahr, von der übergeordneten Macht instrumentalisiert $\mathrm{zu}$ werden bzw. die Instrumentalisierung (unreflektiert) $\mathrm{zu}$ zulassen. Unrühmliche und tragische Beispiele aus der rezenten Geschichte, etwa die Umsetzung von Euthanasieprogrammen, liefern beklemmende Zeugnisse dieser Prozesse und belegen das Gefahrenpotenzial. Weniger dramatisch wirkende, aber inhaltlich bedeutsame Beispiele aus heutiger Zeit beinhalten unter anderem die Realisierung ökonomisch motivierter Entscheidungen, die nicht primär das Wohl des einzelnen Patienten als Maxime betrachten. Das gleichzeitige Gefühl von Macht und Stärke sowie Ohnmacht und Schwäche gehört zu den zentralen Spannungsfeldern der ärztlichen Tätigkeit und das Bio-Macht-Konzept von Foucault liefert ein interessantes Interpretationsfeld dafür.

Foucault befasste sich mit verschiedenen Ausdrucks- und Ausprägungsformen der Macht, unter anderem mit der sogenannten Disziplinarmacht, die in disziplinierenden Gesellschaften durch Mikropraktiken von Institutionen (auch Krankenhäusern) wirksam wird und ein systemkonformes Anpassen des Individuums ermöglicht $[5,10,11]$. Anhand der Überlegungen von Foucault wird deutlich, dass der Umgang mit Macht und deren Auswirkungen ein wichtiges Reflexionsfeld für die Supervision ärztlicher Führungskräfte darstellt, die über andere Ärztinnen und Ärzte, über Patientinnen und Patienten, im akademischen Setting über Studierende und aufgrund ihrer Stellung in der Gesellschaft auch über diese Macht ausüben. Der produktive und verantwortungsvolle Umgang mit dem Komplex Wissen und Macht bringt große Herausforderungen mit sich, denn er erfordert nicht nur ein Bewusstsein über die gestalterischen Möglichkeiten, die den positiven und motivierenden Effekt von Macht widerspiegeln, sondern setzt auch eine reflektierte Betrachtung der destruktiven Möglichkeiten, d. h. des potenziellen Missbrauchs von Macht, voraus. 
Die im Rahmen von machtassoziierten Disziplinierungsprozessen stattfindende Form der Erfahrung bezeichnete Foucault als Disziplinarerfahrung. Diese entsteht als Reflexionsprozess von präexistentem Wissen und liefert keine Impulse für innovatives Denken. Erfahrung wird als Pore eines Individuums verstanden, durch die machtvolles Wissen eindringt und formgebend wirkt [11]. Dem gegenüber stellte er produktive und initiative Erfahrung als allgemeine Einsicht eines anonymen Subjekts der Geschichte dar und argumentierte, dass ein allgemeines Subjekt der Geschichte unabhängig Erfahrung machen und die Geschichte bewegen würde [8]. Er spricht von der „Kontinuität kollektiver Erfahrung “, die bestimmte, in historischen Quellen dokumentierte Effekte in der Organisation gesellschaftlicher Realität erzeugt. Kollektive Erfahrung wird dort zur praktischen Wirklichkeit, wo sie mit der Norm, d. h. dem Wertekatalog der Zeit, übereinstimmt oder diesen selbst produziert. Im Sinne der kollektiven Erfahrung hat sich der leitende Arzt im Krankenhaus zum (Allein-)Herrscher erhoben. Er ist das Gesetz und gleichzeitig der liebende Vater, zu dem die ihm anvertrauten Kinder in Dankbarkeit und Ehrfurcht vor seiner heilenden Wirkung und seinem Wissen hinaufsehen. Er ist das Ebenbild der Norm geworden und verfügt über die Macht, Patientinnen und Patienten und andere in der Hierarchie

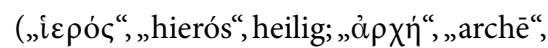
Führung, Herrschaft) nachgeordneten Personen dieser Norm entsprechend zu disziplinieren.

In seinen späteren Werken bezog sich Foucault auf die griechisch-römische Antike und machte damit die (Nach-)Wirkungen aus dieser Epoche für unsere Zeit deutlich. Er postulierte, dass das Individuum auf sich selbst Praktiken anwendet, um bestimmte Ziele zu erreichen, die im Zusammenhang mit seiner historisch und gesellschaftlich spezifischen Verortung stehen, also eine Konsequenz der Macht sind, die im alltäglichen Leben spürbar ist [10]. Die Kontinuität kollektiver Erfahrung und des geschichtlich entstandenen Unbewussten haben im Zusammenspiel mit den kulturhistorischen Grundlagen und Narrativen aus der Antike dem ärztlichen Beruf einen götterähnlichen Status verliehen, dessen Nachwehen in der Perzeption der eigenen Macht zu finden sind.

Auch Pierre Bourdieu (1930-2002) befasste sich in seinen sozialanthropologischen Studien mit Fragen von Macht und Herrschaft und prägte den Begriff symbolische Macht, unter der er eine sanfte und unmerkliche Gewalt verstand, die über symbolische Wege der Kommunikation ausgeübt wird und von den Betroffenen als legitim und wünschenswert akzeptiert wird. Der symbolischen Macht stellte er die materielle Macht gegenüber, die über ökonomische, kulturelle und soziale Faktoren als objektive Machtform in Erscheinung tritt. Die Anerkennung der unterschwellig agierenden symbolischen Macht resultiert nicht aus einem bewussten Entscheidungsprozess, sondern ist im Körper der Akteure präformiert bzw. integriert $[12,13]$. Das lässt die Wahrnehmung der herrschenden sozialen Ordnung als natürlich erscheinen und ermöglicht damit die Verkennung ihres willkürlichen Charakters. Dieser Glaube, den Bourdieu mit dem Begriff Doxa beschrieb, ermöglicht die unbewusste und unreflektierte Inkorporierung der Macht [14]. In modernen Gesellschaften verfügt nach Bourdieu vor allem der Staat über diese symbolische Macht und ersetzt damit die Ausübung physischer Gewalt sowie offen gezeigter politischer Unterdrückung. Die von Bourdieu aufgezeigten Prinzipien und Zusammenhänge könnten in Abhängigkeit von den politischen Umständen durchaus dramatische Erscheinungsformen annehmen. Im Kontext der gesellschaftlichen Position von Ärzten, insbesondere jenen in Führungspositionen, könnten mit diesen Theorien die im Zusammenhang mit dem Terminus Bio-Macht bereits angeführten Spannungsfelder nachvollzogen werden: Die ärztliche Ohnmacht angesichts der übergeordneten symbolischen und materiellen Macht steht der eigenen Macht innerhalb der vorherrschenden hierarchischen Systeme gegenüber. Bourdieu postulierte, dass die soziale Ordnung einer permanenten Verinnerlichung unterliegt und die starren Strukturen der sozialen Welt die Denk- bzw. Handlungsoptionen restringieren [12]. Die klassenspezi- fischen Prägungen, die inkorporierte Geschichte, bestimmen das Wahrnehmen, Denken und Handeln [15]. Die historischen Grundlagen, die als inkorporierte Geschichte unbewusst und unsichtbar präsent sind (etwa die Selbstwahrnehmung als Halbgott in Weiß), stellen eine Prämisse für die Wirkung symbolischer Macht dar. Um diesen Zusammenhang sichtbar zu machen, muss das geschichtlich entstandene Unbewusste [12] aufgezeigt werden, um einen reflektierten Umgang mit der eigenen inkorporierten Geschichte zu ermöglichen, die für die Etablierung des Habitus wesentlich ist. Unter Einbeziehung des Geschichtsaspektes stellt der Habitus nach Bourdieu eine einverleibte, zur Natur gewordene und damit als solche vergessene, verinnerlichte Geschichte dar: „[...] das, was ich einen Habitus nenne, ist eine inkorporierte Geschichte, eine Körper gewordene Geschichte, eingeschrieben in das Gehirn, aber auch in die Falten des Körpers, die Gesten, die Sprechweisen, den Akzent, in die Aussprache, die Tricks, in alles was wir sind. Diese inkorporierte Geschichte ist der Ursprung, von dem aus wir antworten“ [15].

Nach Bourdieu stellt der Habitus demnach eine unbewusst zur Natur gewordene, verinnerlichte Geschichte dar, die den Verhaltens- und Handlungsspielraum determiniert. Der ärztliche Habitus, insbesondere jener von Führungskräften, der zu einem wesentlichen Teil auf einer inkorporierten Geschichte beruht, die das Narrativ von göttergleicher (All-)Macht und Allwissenheit beinhaltet, beeinflusst maßgeblich auch die Einstellung und den Zugang dieser Berufsgruppe zur Supervision. Die angeführten Arbeiten von Michel Foucault und Pierre Bourdieu haben mit den von ihnen geprägten Begriffen Kontinuität kollektiver Erfahrung, inkorporierte Geschichte, Habitus, Doxa, Bio-Macht, materielle und symbolische Macht theoretische Grundlagen für das Verständnis und die Interpretation der prägenden kulturhistorischen Narrative für das ärztliche Selbstverständnis geliefert. Damit können Prinzipien aufgezeigt werden, welche die Einstellung gegenüber Supervision/Coaching charakterisieren und 
die wichtige Hürden für die offene Annahme des Beratungsangebots darstellen.

Die Nachklänge des von der Antike mitgeformten ärztlichen Habitus, mit der sicherlich im Schwinden begriffenen $\mathrm{Hy}$ bris, dürften die Möglichkeiten supervisorischer Arbeit im ärztlichen Bereich sicherlich noch eine Zeit lang beeinflussen. Da die dem (ärztlichen) Habitus zugrundeliegende inkorporierte Geschichte in der Regel nicht bewusst wahrgenommen wird, ist es nach Bourdieu notwendig, zunächst das geschichtlich entstandene Unbewusste [12] zu entschleiern, bevor eine reflektierte Auseinandersetzung mit dem eigenen beruflichen Selbstverständnis möglich wird. Diese theoretische Analyse von Bourdieu liefert die Einsicht, dass die Unterstützung des Erkenntnisprozesses über die inkorporierte Geschichte [15] bzw. die Kontinuität kollektiver Erfahrung [8] und den daraus resultierenden persönlichen Haltungen und Verhaltensnormen eine Prämisse für die Akzeptanz von Supervision im ärztlichen Bereich darstellt.

\section{Abschließende Anmerkungen}

Die Wissenschaftshistorikerin Margaret W. Rossiter hat im Jahr 1993 den sogenannten Matilda-Effekt postuliert [16], der die systematische Verdrängung und Leugnung des Beitrags von Wissenschafterinnen beschreibt, deren Arbeit häufig ihren männlichen Kollegen zugerechnet wird. Es ist daher vielleicht nicht besonders überraschend, dass fast alle allgemein bekannten Größen der Medizingeschichte Männer waren. Man muss tatsächlich einigen Aufwand betreiben, um Hinweise auf bedeutende Ärztinnen von der Antike bis zur Neuzeit zu finden, die es natürlich auch gegeben hat. Die Stellen im vorliegenden Text, in denen nur die männliche Form Arzt angeführt ist, meinen daher in der Regel Ärztinnen tatsächlich nicht mit, und es ist fraglich, ob die inkorporierte Geschichte von Ärztinnen von den antiken Narrativen ähnlich geprägt werden konnte, wie jene der männlichen Kollegen. Die für supervisorische Arbeit relevanten Unterschiede im gängigen Habitus zwischen Ärzten und Ärztinnen, die im letzteren Fall oftmals durch eine größe- re Offenheit für Supervision/Coaching geprägt sind, konnten in diesem Beitrag aus Platzgründen leider nicht diskutiert werden.

Zum Schluss sollte hervorgehoben werden, dass die präsentierten Thesen natürlich keinesfalls den Anspruch aufalleinige oder absolute Wahrheit erheben. Möglicherweise liefern sie aber nachvollziehbare Erklärungskonzepte für die beobachtete Einstellung zu Supervision bzw. Coaching im ärztlichen Bereich und geben Anregungen zur Reflexion über das eigene ärztliche Selbstverständnis.

\section{Korrespondenzadresse

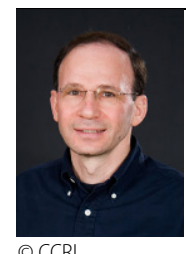 \\ Univ.-Prof. DDr. Thomas Lion, MSc Zimmermannplatz 10/8, 1090 Wien, Österreich Thomas.Lion@ccri.at thomas.lion@ meduniwien.ac.at}

Univ.-Prof. DDr. Thomas Lion Ärztlicher Direktor des Ambulatoriums Labdia Laboratoriumsdiagnostik $\mathrm{GmbH}$, Wien; Leiter der Abteilung für molekulare Mikrobiologie, Forschungsinstitut für krebskranke Kinder, St. Anna Kinderkrebsforschung, Wien; Facharzt für Kinder- und Jugendheilkunde mit Schwerpunkt Hämatoonkologie, St. Anna Kinderspital, Wien; Facharzt für medizinische und chemische Labordiagnostik, Universität Wien; Additiv-Facharzt für Humangenetik; Graduierter Supervisor und Coach (ÖAGG). Doppelstudium Medizin und Sportwissenschaften (Uni Wien) und Doktoratsprogramm an der Naturwissenschaftlichen Fakultät der Karlsuniversität in Prag (Promotionen 1984 und 1995); Postdoc an der University of Chicago und University of Illinois, USA (1986-1988); Masterstudium in Sozialwissenschaften (Uni Wien MSc 2018)

Funding. Open access funding provided by Medical University of Vienna.

\section{Einhaltung ethischer Richtlinien}

Interessenkonflikt. T. Lion gibt an, dass kein Interessenkonflikt besteht.

Dieser Beitrag beinhaltet keine von den Autoren durchgeführten Studien an Menschen oder Tieren.

Open Access Dieser Artikel wird unter der Creative Commons Namensnennung 4.0 International Lizenz (http://creativecommons.org/licenses/by/4.0/deed. de) veröffentlicht, welche die Nutzung, Vervielfältigung, Bearbeitung, Verbreitung und Wiedergabe in jeglichem Medium und Format erlaubt, sofern Sie den/die ursprünglichen Autor(en) und die Quelle ordnungsgemäß nennen, einen Link zur Creative Commons Lizenz beifügen und angeben, ob Änderungen vorgenommen wurden.
Hinweis des Verlags. Der Verlag bleibt in Hinblick auf geografische Zuordnungen und Gebietsbezeichnungen in veröffentlichten Karten und Institutsadressen neutral.

\section{Literatur}

1. Wittich Andrea, Dieterle W (2009) Empirische Supervisionsforschung: Ein Beitrag aus dem Krankenhaus. In: Haubl R, Hausinger B (Hrsg) Supervisionsforschung: Einblicke und Ausblicke. Vandenhoeck \& Ruprecht, Göttingen. ISBN 978-3525-40325-9

2. Becker-Kontio M (2004) Bericht der Projektgruppe "Supervision im Gesundheitswesen" in der Deutschen Gesellschaft für Supervision e. V. In: Becker-Kontio M, Kimmig-Pfeiffer A, Schwennbeck $M-L$, Streitbürger G, Wengelski-Strock S (Hrsg) Supervision und Organisationsberatung im Krankenhaus: Erfahrungen - Analysen - Konzepte (Veröffentlichungen der Deutschen Gesellschaft für Supervision e. V.). Juvent, Weinheim. ISBN 9783-779-91786-1

3. Eichberger Gerd (2013) Was bedeutet Supervision für Führungskräfte im ärztlichen Bereich? Akademikerverlag, Saarbrücken. ISBN 978-3-639-471779

4. Golder W (2007) Hippokrates und das Corpus Hippocraticum, De decenti ornatu. Königshausen u. Neumann, Würzburg. ISBN 978-3-826-03335-3

5. Foucault M (1973) Die Geburt der Klinik: Eine Archäologie des ärztlichen Blicks. Hanser, München. ISBN 978-3-596-27400-0

6. Foucault M (1977) Überwachen und Strafen: Die Geburt des Gefängnisses. Suhrkamp, Frankfurt am Main. ISBN 978-3-518-27784-3

7. Foucault M (1977) Der Wille zum Wissen:Sexualität und Wahrheit 1. Suhrkamp, Frankfurt am Main. ISBN 978-3-518-07470-1

8. Foucault M (2002) Archäologie des Wissens. Suhrkamp, Frankfurt am Main. ISBN 978-3-51827956-4

9. Foucault M (2005) Die Maschen der Macht In: Defert D, Francois E (Hrsg) Analytik der Macht. Suhrkamp, Frankfurt am Main. ISBN 9783518293591

10. Foucault M (1989) Gebrauch der Lüste: Sexualität und Wahrheit 2. Suhrkamp, Frankfurt am Main. ISBN 978-3-518-28317-2

11. Foucault M (1973) Wahnsinn und Gesellschaft. Suhrkamp, Frankfurt am Main. ISBN 978-3-51827639-6

12. Bourdieu $P$ (2005) Die männliche Herrschaft. Suhrkamp, Frankfurt am Main. ISBN 978-3-51829631-8

13. Bourdieu $P$ (2005) Die verborgenen Mechanismen der Macht. VSA, Hamburg. ISBN 978-3-879-75605-

14. Wayand G, Bourdieu P (1998) Das Schweigen der Doxa aufbrechen. In: Imbusch P (Hrsg) Macht und Herrschaft: Sozialwissenschaftliche Konzeptionen und Theorien. Springer VS, Wiesbaden. ISBN 9783-663-10691-3

15. Bourdieu $P$ (2001) Wie die Kultur zum Bauern kommt. VSA, Hamburg. ISBN 978-3-879-75803-6

16. Rossiter Margaret W (1993) The Matilda effect in science. In: Social studies of science, Bd. 23. SAGE, London, S325 (http://dispatch.opac.dnb.de/) 